\title{
Ionospheric anomaly due to seismic activities-III: correlation between night time VLF amplitude fluctuations and effective magnitudes of earthquakes in Indian sub-continent
}

\author{
S. Ray $^{1}$, S. K. Chakrabarti ${ }^{1,2}$, S. K. Mondal ${ }^{1}$, and S. Sasmal ${ }^{1}$ \\ ${ }^{1}$ Indian Centre for Space Physics, 43 Chalantika, Garia Station Road, Kolkata 700084, India \\ ${ }^{2}$ S. N. Bose National Centre for Basic Sciences, JD Block, Salt lake, Kolkata 700098, India
}

Received: 10 April 2011 - Revised: 25 July 2011 - Accepted: 12 September 2011 - Published: 10 October 2011

\begin{abstract}
We present the results of an analysis of yearlong (2007) monitoring of night time data of the VLF signal amplitude from the Indian Navy station VTX at $18.2 \mathrm{kHz}$, received by the Indian Centre for Space Physics, Kolkata. We analyzed this data to find out the correlation, if any, between night time amplitude fluctuation and seismic events. We found, analyzing individual cases (with magnitudes $>5$ ) as well as statistical analysis (of all the events with effective magnitudes greater than 3.5), that night time fluctuation of the signal amplitude has the highest probability to be beyond the $2 \sigma$ level about three days prior to seismic events. Thus, the night time fluctuation could be considered as a precursor to enhanced seismic activities.
\end{abstract}

\section{Introduction}

It has been pointed out almost two decades ago that night time radio signals show anomalous fluctuations several days before earthquakes (Gokhberg et al., 1989; Gufeld et al., 1992; Rozhnoi et al., 2004; Maekawa et al., 2006; Horie et al., 2007; Rozhnoi et al., 2009; Kasahara et al., 2010; Hayakawa et al., 2010). There are several other methods such as the terminator time method (Hayakawa et al., 1996; Molchanov et al., 1998; Molchanov and Hayakawa, 1998, 2008; Hayakawa and Molchanov, 2000; Chakrabarti et al., 2007; Sasmal et al., 2009 (Paper I); Molchanov, 2009) and the D-layer preparation time and the D-layer disappearance time (Chakrabarti et al., 2007, 2010 (Paper II)), which also lend some support to the hypothesis that ionospheres are perturbed by seismic activities well before the earthquake is actually felt. Some theoretical models have been advanced and numerical simulations carried out. Analyzing observations

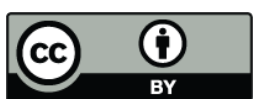

Correspondence to: S. K. Chakrabarti (chakraba@bose.res.in) around 2005 earthquakes in Greece, Contadakis et al. (2008) found that the total electron content (TEC) may have varied over a month before the seismic activity. Naaman et al. (2001) studied the correlation among the ionospheric total electron content and geomagnetic fields that are associated with strong earthquakes. Some of the strong contenders in understanding the anomalies are the so-called acoustic models (Liperovsky et al., 1997) and the Lithosphere-Ionosphere coupling model (see Pulinets and Ouzounov, 2011 and references therein; Liperovskaya et al., 2010; Meister et al., 2011). The turbulence generated in the ionosphere due to Atmospheric Gravity Waves (the so-called AGW model) may also be one of the causes of the lithosphere-ionosphere coupling (Molchanov, 2009). Using some simple models, it was shown that a lowering of the ionosphere by $1-2 \mathrm{~km}$ could mimic the terminator time shift (Hayakawa et al., 1996; Molchanov et al., 1998). However, others (Rodger et al., 1999; Soloviev and Hayakawa, 2002; Soloviev et al., 2004) showed that it would require a lowering of the ionosphere by 4-11 km, which would have other observational effects as in the cases of solar flares (Rodger et al., 1999; Soloviev and Hayakawa, 2002; Soloviev et al., 2004). The conclusions of these authors were that the observed shifts in terminators are possible only if the propagation path is short $(<2500$ $3000 \mathrm{~km}$ ) (Yoshida et al., 2008). However, the subject is still in a nascent stage, and the relationship between such anomalies and seismicity is poorly understood up to now (e.g., Clilverd et al., 1999; Rodger et al., 1999; Hayakawa et al., 2002).

The Indian Centre for Space Physics has been monitoring the VLF signals from various stations to look for such ionospheric anomalies and to check if any correlations could be found among them. The data was procured by the AWESOME (Atmospheric Weather Electromagnetic System for Observation Modeling and Education) receiver made by Stanford University VLF Group. Our study is particularly important since the Indian sub-continent is an earthquake prone region and any reasonable prediction of earthquakes

Published by Copernicus Publications on behalf of the European Geosciences Union. 
could save millions of lives. In the present paper, we report results of our analysis of the data for year 2007. We find that three days prior to major seismic activities, the night time fluctuation of the signal amplitude is the most significant. This is found to be the case for many individual earthquakes. At the same time, when we compute the effective magnitudes of the collection of earthquakes on each day and correlate our computations with the night time fluctuations, we find that this statistical correlation also peaks three days prior to the event date. This result is consistent with the typical 1-5 days of precursor time found in other methods (Molchanov and Hayakawa, 2008; Hayakawa, 2009; Kasahara et al., 2010; Liperovskaya et al., 2008, 2010, 2011; Contadakis et al., 2008). In the next section, we present the methodology of our analysis, namely, how we obtained the fluctuations from the mean on a daily basis, and how we obtained the effective magnitudes. In Sect. 3, we discuss our results. Finally, in Sect. 4, we present the concluding remarks.

\section{Methodology of our analysis}

Our receiving station is situated at the Indian Centre for Space Physics (ICSP) located in the south side of Kolkata $\left(22^{\circ} 34^{\prime} \mathrm{N}, 88^{\circ} 34^{\prime} \mathrm{E}\right)$ city. We use the AWESOME receiver, which receives signals from several stations. In particular, we concentrate on the Indian Navy VLF transmitter station VTX, which is located at Vijayanarayanam $\left(8^{\circ} 26^{\prime} \mathrm{N}\right.$, $77^{\circ} 44^{\prime} \mathrm{E}$ ). This station transmits the VLF signal at $18.2 \mathrm{kHz}$. In an AWESOME receiver, the antenna has two cross-loops capable of detecting fluctuating magnetic field components in the VLF signals. After receiving the data by this antenna, first it is pre-amplified and time stamped by a GPS unit. The data is automatically stored in the computer. As a backup, we also have our Gyrator-III type receiver capable of tuning into a single transmitter. For this receiver there is a single loop antenna. However, in the analysis below we used only AWESOME data.

For the present analysis, we consider only the data of local (IST $=\mathrm{UT}+5: 30 \mathrm{~h}$ ) night time beginning at $19: 30 \mathrm{~h}$ (14:00 UT) and ending at 04:30 h (23:00 UT) of the (local) next day with a one hour gap just prior to midnight for data analysis. We stayed away from sunrise and sunset terminators by at least an hour to avoid contamination from the D-layer formation or disappearance effects. Using the data set on each day (denoted by $i$ ), we compute the mean and the standard deviation $\left(\Sigma_{i}\right)$, which is our fluctuation measure. In the lower panel of Fig. 1, we plot $\Sigma_{i}$ (marked with "+" signs) in $\mathrm{dB}$ as a function of $i$. In order to quantify some fluctuations as anomalous, we need to know what the mean and standard deviation of quiet day fluctuations are. We are assuming that the following is a reasonable procedure to define the mean and the standard deviation of quiet days: we first take the mean and the standard deviation $\sigma$ out of all $\Sigma_{i}$ s and eliminate those fluctuations which are beyond

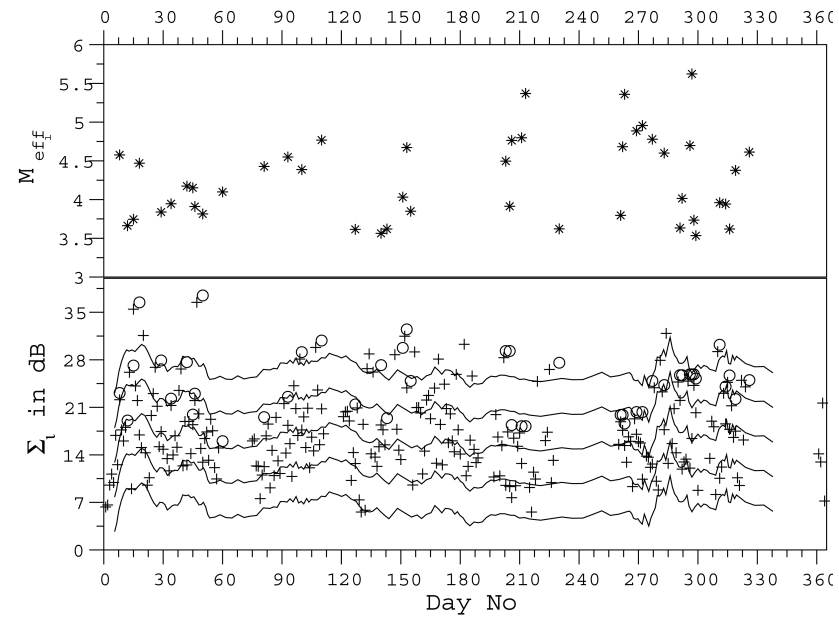

Fig. 1. In the upper panel we plot the effective magnitude (>3.5) of the seismic event on each day. The lower panel shows the variation of $\Sigma_{i}$ (marked with a "+" sign), the standard deviation of the nighttime fluctuation of signal amplitude on $i$-th day, as a function of day number $i$ for the year 2007. The curve in the center is the mean drawn through these $\Sigma_{i}$ and the curves above and below are for $\pm \sigma, \pm 2 \sigma$ variations around the mean. The circles represent the earthquakes having effective magnitudes $M_{\text {eff }_{i}}>3.5$ on each day (shifted suitably) just to show the possible association of the earthquake with an observation.

the $2 \sigma$ level. This way we remove "significantly" anomalous data from our collection of $\Sigma_{i}$ s to obtain the profile of the mean fluctuation throughout the year, possibly in the absence of seismic events. After this, we compute the mean and then compute the standard deviation $\sigma$ once more. This $\sigma$ would naturally be smaller. The solid curve in the center represents the mean curve. The solid curves above and below are at $\pm 1 \sigma$ and $\pm 2 \sigma$ levels, respectively. We clearly see many night time fluctuations of the amplitudes which are beyond the $2 \sigma$ level. On certain days, there were seismic activities. The upper panel shows the effective magnitudes $M_{\text {eff }}$ by a star symbol (*). These are obtained by using a procedure described later in this section. In the lower panel we show the activities by open circles. They were placed adjacent to the data suspected to be associated with that quake, just for reference. However, true association would automatically be determined from computation of the correlations (see below). The effective magnitudes $\left(M_{\mathrm{eff}_{i}}\right)$ of the activities on each day were computed using the following procedure: We consider the seismic events which occurred in the neighborhood of the GCP (Great Circle Path defined as the shortest path between two geo-coordinates.) between the transmitter and the receiver and compute the effective magnitudes. For this purpose, we collect the latitudes and longitudes of the earthquakes, their magnitudes, the depths of the epicenters, etc. from the Indian Meteorological Department 
(http://www.imd.gov.in). We calculate the energy of the earthquake by using the equation $\log _{10} E=4.4+1.5 M_{\mathrm{S}}$, (for earthquakes $M<5$ ) and $\log _{10} E=5.24+1.44 M_{\mathrm{s}}$, (for earthquakes $M>5$ ), where $E$ - energy of the earthquake in Jules and $M_{\mathrm{S}}$ - surface wave magnitude (Lowrie, 2007). These $M_{\mathrm{S}}$ were converted to $M$ by the formula: $M_{\mathrm{s}}=-3.2+1.45 M$ (Tobyas and Mittag, 1991). These are empirical relations and thus do not match exactly for $M=5.0$. However, this does not affect the result. On days when more than one earthquake occurs, we use the following procedure to obtain the effective magnitude $M_{\text {eff }}$ for that date: To obtain the energy deposition by the earthquakes at middle point (i.e., sub-reflection point [SRP] of the first hop) between the transmitter and the receiver, we divide the energies of the earthquakes by the GCP distance between the mid reflection point and the epicenter of the earthquakes. Here we assume that the wave is propagating as a Rayleigh wave, though for earthquakes with epicenters deep inside, there may be deviation from this consideration. After adding all of these energy contributions on a given day, and using the above formulas in reverse, we calculate the $M_{\mathrm{eff}_{i}}=M_{\mathrm{s}}$ for each day. Finally, we converted this $M_{\mathrm{S}}$ into $M$. To avoid clumsiness, we choose only the earthquakes having effective magnitude $>3.5$. As discussed above, this effective magnitude is a measure of the amount of energy deposition at the mid reflection point, and its major contribution comes from the strongest seismic activities.

It is to be noted that Fig. 1 is for illustration purpose only. While computing the correlation between the standard deviation and the effective magnitude, we used all the data and not just those which appear to be anomalous. Second, we do not consider only those earthquakes that are within a few Fresnel zones (Molchanov and Hayakawa, 1998; Rozhnoi et al., 2004). Computation of the Fresnel zones from the epicenters indicate that the Fresnel zone radii are only about a hundred kilometers. This is because in the statistical analysis, we are considering the correlation with the effective magnitude arising out of all earthquakes occuring within $3000 \mathrm{~km}$ of the SRP on a given day, and not just a single earthquake.

\section{Correlations of the night time fluctuations and seismic activities}

In Fig. 2, we present two typical examples of the night time amplitude variation of the VTX signal as a function of time (UT). To cross-check, we calculate the mean night time VTX signal amplitude in Kolkata using LWPC software and find it to be $\sim 80 \mathrm{~dB}$. This is consistent with our observation. The "normal" data (dashed curve) of 15 February 2007 with fluctuation below $1 \sigma$ (Fig. 1) is compared with "anomalous" data (solid curve) of 16 February 2007 with higher night time fluctuation. On 19 February 2007 there was a moderately strong earthquake $(M=5.4)$ at Southern Sumatra, Indonesia. The anomalous fluctuation of 16 February 2007 could be the precursor of this earthquake.

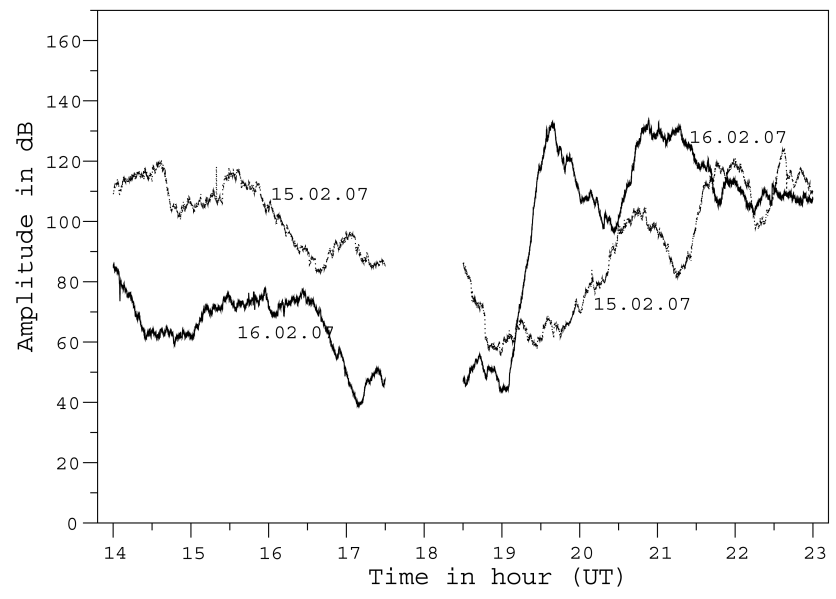

Fig. 2. An example of a quiet night time (15 February 2007) data as compared with an anomalously fluctuating night time (16 February 2007) data. High fluctuations in the signal amplitude on the 16 February 2007 is evident.
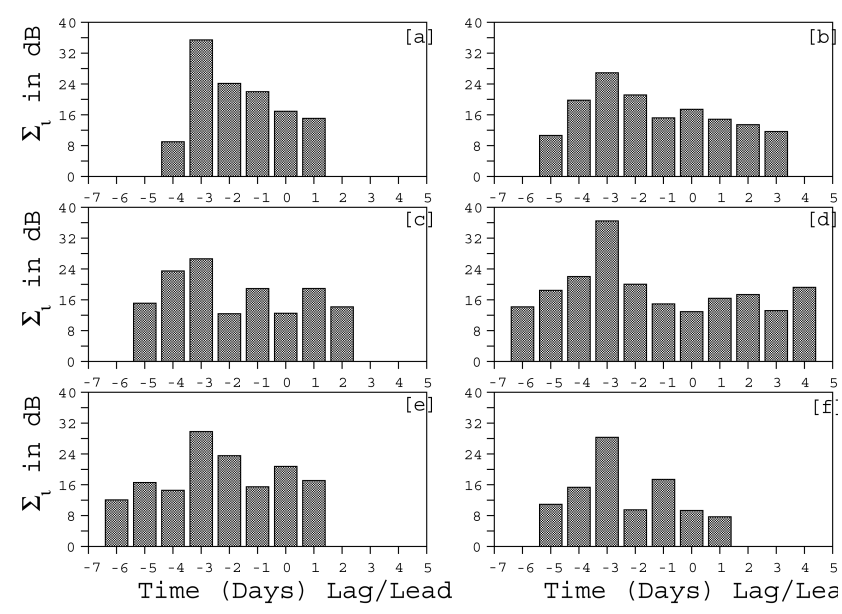

Fig. 3. Examples of some cases where the fluctuation measure $\Sigma_{i}$ became anomalously high three days prior to the seismic activities. (a) 18 January $2007(M=5.8)$ at Southern Sumatra, Indonesia; (b) 29 January $2007(M=5.2)$ at north of Camorta, Nicobar; (c) 11 January $2007(M=5.6)$ at off-coast of Sumatra; (d) 19 January $2007(M=5.4)$ at southern Sumatra; (e) 20 April 2007 ( $M=$ 6.2) at Southwestern Ryukyu Islands, Japan and (f) 24 July 2007 $(M=5.4)$ at northern Sumatra.

First we show that when we consider a case-by-case basis, many of the strong quakes show a correlation with $\Sigma_{i}$. In Fig. 3a-f, we plot $\Sigma_{i}$ s for a few days around six prominent earthquakes: (a) 18 January $2007(M=5.8)$ at Southern Sumatra, Indonesia; (b) 29 January $2007(M=5.2)$ at north of Camorta, Nicobar; (c) 11 February $2007(M=5.6)$ at offcoast of Sumatra; (d) 19 February $2007(M=5.4)$ at southern Sumatra; (e) 20 April $2007(M=6.2)$ at Southwestern 


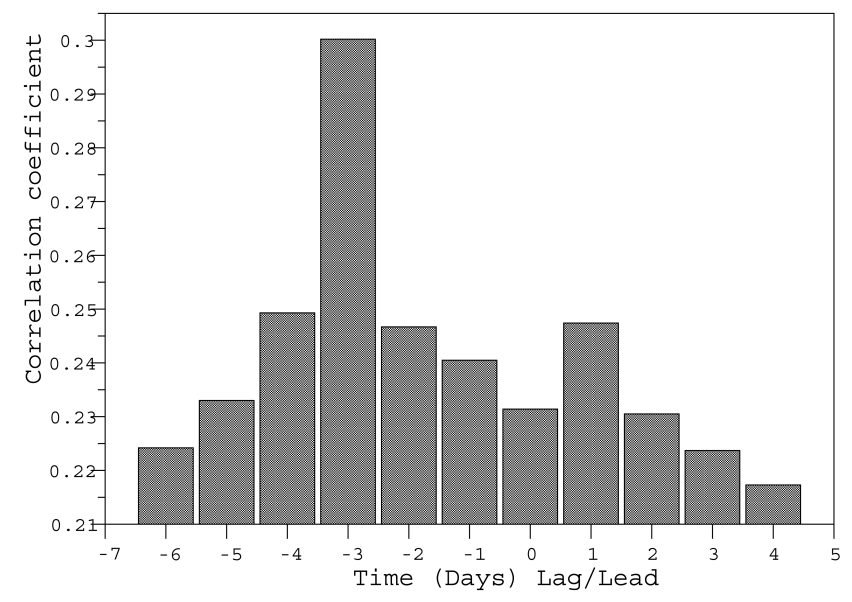

Fig. 4. Correlation between the effective magnitude $M_{\mathrm{eff}_{i}}$ of the earthquake at the middle point between the transmitter and the receiver, and the deviation of the fluctuation measure $\Sigma_{i}$. Note that the correlation peaks three days before the event days.

Ryukyu Islands, Japan; and (f) 24 July 2007 ( $M=5.4)$ at northern Sumatra. In all of these cases, we find that three days prior to the earthquake, $\Sigma_{i}$ became around $30 \mathrm{~dB}$ or more. Clearly, this is not the case for all the earthquakes. That is why we carry out the statistical analysis. In Fig. 4, we plot the correlation coefficients between the deviation of the $\Sigma_{i}$ and $M_{\text {eff }_{i}}$. The histograms are drawn at one day interval. Here too, we find that the peak of the correlation is located at three days before the event day. The peak is not unity, since the correlation is not certain. There are significant correlations in other nearby days also. However, we could interpret these coefficients as the un-normalized probability for seismic events after the corresponding number of days. Our prediction of an earthquake within three days is in line with the 2 to 5 day pre-cursors discussed in other works (Rozhnoi et al., 2004; Maekawa et al., 2006; Molchanov and Hayakawa, 2008; Kasahara et al., 2010).

In order to see if the deviation of $\Sigma_{i}$ from its mean value is related to the effective magnitude of the earthquake, in Fig. 5 we plot the deviation of $\Sigma_{i}$ of all available data exactly three days before seismic events. We clearly note that for $M_{\text {eff }_{i}}<1.5$ the deviation is negligible. However, beyond that the deviation generally increases with $M_{\text {eff }_{i}}$. The solid line is the best fit of all the points for which the earthquakes are at low depths. The grey line is the best fit of all the points for which earthquakes are at high depths. Considerable scatter on both the sides of the lines are seen.

\section{Concluding remarks}

It has long been recognized that the prediction of earthquakes is one of the most difficult tasks faced by the scientific

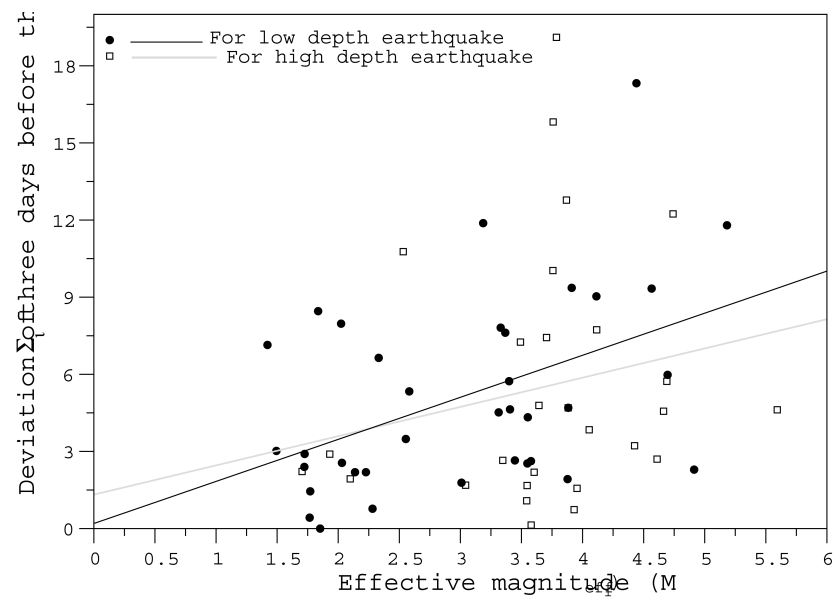

Fig. 5. Variations of the deviation of $\Sigma_{i}$ from the mean value as a function of the effective magnitudes of the earthquake are plotted. Here we plot the deviation of $\Sigma_{i}$ of all available data exactly three days before seismic events. The dark circles are for epicenters less than $30 \mathrm{~km}$ and open boxes are for epicenters above $30 \mathrm{~km}$. The solid line is the best fit of all the points for which the earthquakes are at low depths (i.e. epicenters less than $30 \mathrm{~km}$ ). The grey line is the best fit of all the points for which earthquakes are at high depths (i.e., epicenters above $30 \mathrm{~km}$ ). For $M_{\mathrm{eff}_{i}}<1.5$, the deviation is negligible and it monotonically increases with the effective magnitude. A considerable scatter is seen on both sides of the line fitted taking all the points.

community throughout the world. Any new input, findings, or correlations could add to our understanding. While the night time fluctuations were thought to be precursors of an earthquake event, very few studies have been made on this phenomenon (Rozhnoi et al., 2004; Maekawa et al., 2006; Molchanov and Hayakawa, 2008; Kasahara et al., 2010). In this paper, we present the analysis of our results. Unlike others, we do not quantify the fluctuations by Fourier analysis, but we simply measure the deviation from the mean signal amplitude. We show both by the case-by-case analysis of several major earthquakes and by a statistical analysis of all the events in a year that there are hints of significantly large fluctuations in the night time data three days prior to earthquake activities. There are significant fluctuations before and after, since the correlation coefficient variation with time lag does not follow a sharp curve. Figure 4 can thus be interpreted as un-normalized probability of an earthquake occurrence within next few days. However, the appearance of the peaks seems to be robust in both the case-by-case analysis and in the statistical analysis. Because there are seismic events every after few days (especially when strong after shocks are present), it is difficult to compute the correlations for a very long time since the effect would be due to multiple seismic events. We believe that our results may be treated as the strongest ever evidence of correlations between 
the ionospheric anomalies and the seismic events. However, pre-event pinpointing the location of the epicenter is still a distant goal. In future, we will analyze data of other stations and from the fluctuations of amplitudes from several stations at a given time, it may be easier to narrow down the location of future seismic activities.

Acknowledgements. S. Ray and S. Sasmal thank a RESPOND project of ISRO, and S. K. Mondal thanks CSIR Junior Research Fellowship.

Edited by: M. E. Contadakis

Reviewed by: two anonymous referees

\section{References}

Chakrabarti, S., Sasmal, S., Saha, M., Khan, M., Bhowmik, D., and Chakrabarti, S. K.: Unusual behaviour of D-region ionization time at $18.2 \mathrm{kHz}$ during seismically active days, Indian J. Phys., 81, 531-538, 2007.

Chakrabarti, S. K., Sasmal, S., and Chakrabarti, S.: Ionospheric anomaly due to seismic activities - Part 2: Evidence from Dlayer preparation and disappearance times, Nat. Hazards Earth Syst. Sci., 10, 1751-1757, doi:10.5194/nhess-10-1751-2010, 2010.

Clilverd, M. A., Rodger, C. J., and Thomson, N. R.: Investigating seismoionospheric effects on a long subionospheric path, J. Geophys. Res., 104, 28171-28179, 1999.

Contadakis, M. E., Arabelos, D. N., Asteriadis, G., Spatalas, S. D., and Pikridas, C.: TEC variations over the Mediterranean during the seismic activity period of the last quarter of 2005 in the area of Greece, Nat. Hazards Earth Syst. Sci., 8, 1267-1276, doi:10.5194/nhess-8-1267-2008, 2008.

Gokhberg, M. B., Gufeld, I. L., Rozhnoy, A. A., Marenko, V. F., Yampolsky, V. S., and Ponomarev, E. A.: Study of seismic influence on the ionosphere by super long-wave probing of the Earth-ionosphere waveguide, Phys. Earth Planet. Inter., 57, 6467, 1989.

Gufeld, I. L., Rozhnoi, A. A., Tyumensev, S. N., Sherstuk, S. V., and Yampolsky, V. S.: Radio wave field disturbances prior to Rudbar and Rachinsk earthquakes, Phys. Solid Earth, 28, 267-270, 1992.

Hayakawa, M.: Lower ionospheric perturbations associated with earthquakes, as detected by subionospheric VLF/LF radio waves, in: Electromagnetic Phenomena Associated with Earthquakes, edited by: Hayakawa, M., Transworld Research Network, Trivandrum(India), 137-185, 2009.

Hayakawa, M., Molchanov, O. A., Ondoh, T., and Kawai, E.: The precursory signature effect of the Kobe earthquake on VLF subionospheric signals, J. Comm. Res. Lab., Tokyo, 43, 169180, 1996.

Hayakawa, M. and Molchanov, O. A.: Effect of earthquakes on lower ionosphere as found by subionospheric VLF propagation, Adv. Space Res., 26, 8, 1273-1276, 2000.

Hayakawa, M., Molchanov, O. A., Shima, N., Shvets, A. V., and Yamamoto, N.: Wavelet analysis of disturbances in subionospheric VLF propagation correlated with earthquakes, in: Seismo Electromagnetics: Lithosphere Atmosphere-Ionosphere Couplings, edited by: Hayakawa, M. and Molchanov, O. A., (TERRAPUB, Tokyo, Japan), 223-228, 2002.
Hayakawa, M., Kasahara, Y., Nakamura, T., Muto, F., Horie, T., Maekawa, S., Hobara, Y., Rozhnoi, A. A., Solivieva, M., and Molchanov, O. A.: A statistical study on the correlation between lower ionospheric perturbations as seen by subionospheric VLF/LF propagation and earthquakes, J. Geophys. Res., 115, 09305, doi:10.1029/2009JA015143, 2010.

Horie, T., Maekawa, S., Yamauchi, T., and Hayakawa, M.: A possible effect of ionospheric perturbations associated with the Sumatra earthquake, as revealed from subionospheric very-lowfrequency (VLF) propagation (NWC-Japan), Int. J. Remote Sens., 28, 3133-3139, 2007.

Kasahara, Y., Muto, F., Horie, T., Yoshida, M., Hayakawa, M., Ohta, K., Rozhnoi, A., Solovieva, M., and Molchanov, O. A.: On the statistical correlation between the ionospheric perturbations as detected by subionospheric VLF/LF propagation anomalies and earthquakes, Nat. Hazards Earth Syst. Sci., 8, 653-656, doi:10.5194/nhess-8-653-2008, 2008.

Kasahara, Y., Muto, F., Hobara, Y., and Hayakawa, M.: The ionospheric perturbations associated with Asian earthquakes as seen from the subionospheric propagation from NWC to Japanese stations, Nat. Hazards Earth Syst. Sci., 10, 581-588, doi:10.5194/nhess-10-581-2010, 2010.

Liperovskaya, E. V., Bogdanov, V. V., Biagi, P.-F., Meister, C.-V., Liperovsky, V. A., and Hoffmann, D. H. H.: Day-time variations of foE and foF2 connected to earthquakes, EGUGA, 12, 2542, 2010.

Liperovskaya, E. V., Bogdanov, V. V., Biagi, P.-F., Meister, C.V., and Liperovsky, V. A.: Daytime variations of foE connected to earthquakes, Nat. Hazards Earth Syst. Sci., 11, 1807-1812, doi:10.5194/nhess-11-1807-2011, 2011.

Liperovsky, V. A., Meister, C.-V., Schlegel, K., and Haldoupis, C.: Currents and turbulence in and near mid-latitude sporadic Elayers caused by strong acoustic impulses, Ann. Geophys., 15, 767-773, doi:10.1007/s00585-997-0767-x, 1997.

Liperovsky, V. A., Pokhotelov, O. A., Meister, C.-V., and Liperovskaya, E. V.: On recent physical models of lithosphereatmosphere-ionosphere coupling before earthquakes, Geomagn. Aeronomy, 48, 795-806, 2008.

Lowrie, W.: Fundamentals of Geophysics, Cambridge University press, 2007.

Maekawa, S., Horie, T., Yamauchi, T., Sawaya, T., Ishikawa, M., Hayakawa, M., and Sasaki, H.: A statistical study on the effect of earthquakes on the ionosphere, based on the subionospheric LF propagation data in Japan, Ann. Geophys., 24, 2219-2225, doi:10.5194/angeo-24-2219-2006, 2006.

Meister, C.-V., Mayer, B., Dziendziel, P., Flbert, F., Hoffmann, D. H. H., and Liperovsky, V. A.: On the acoustic model of lithosphere-atmosphere-ionosphere coupling before earthquakes, Nat. Hazards Earth Syst. Sci., 11, 1011-1017, doi:10.5194/nhess-11-1011-2011, 2011.

Molchanov, O. and Hayakawa, M.: Seismo-electromagnetics and related phenomena: History and latest results, TERRAPUB, Tokyo, 190, 2008.

Molchanov, O.: Lithosphere-atmosphere-ionosphere coupling due to seismicity, in: Electromagnetic phenomena associated with earthquakes, edited by: Hayakawa, M., 254-279, Transworld Research Network, Trivandrum (India), 2009.

Molchanov, O. A. and Hayakawa, M.: Subionospheric VLF signal perturbations possibly related to earthquakes, J. Geophys. Res., 
103, 17489-17510, 1998.

Molchanov, O. A., Hayakawa, M., Ondoh, T., and Kawai, E.: Precursory effects in the subionospheric VLF Signals for the Kobe earthquake, Phys. Earth Planet. Inter., 105, 239-248, 1998.

Naaman, Sh., Alperovich, L. S., Wdowinski, Sh., Hayakawa, M., and Calais, E.: Comparison of simultaneous variations of the ionospheric total electron content and geomagnetic field associated with strong earthquakes, Nat. Hazards Earth Syst. Sci., 1, 53-59, doi:10.5194/nhess-1-53-2001, 2001.

Pulinets, S. and Ouzounov, D.: Lithosphere-AtmosphereIonosphere coupling (LAIC) model - A united concept for earthquake precursors validation, Asian J. Earth Sci., 41, 371-382, 2011

Rodger, C. J., Clilverd, M. A., and Thomson, N. R.: Modeling of subionospheric VLF signal perturbations associated with earthquakes, Radio. Sci., 34, 1177-1185, 1999.

Rozhnoi, A., Solovieva, M. S., Molchanov O. A., and Hayakawa, M.: Middle latitude LF (40 kHz) phase variations associated with earthquakes for quiet and disturbed geomagnetic conditions, Phys. Chem. Earth, 29, 589-598, 2004.

Rozhnoi, A., Solovieva, M., Molchanov, O., Schwingenschuh, K., Boudjada, M., Biagi, P. F., Maggipinto, T., Castellana, L., Ermini, A., and Hayakawa, M.: Anomalies in VLF radio signals prior the Abruzzo earthquake $(M=6.3$ ) on 6 April 2009, Nat. Hazards Earth Syst. Sci., 9, 1727-1732, doi:10.5194/nhess-9-17272009, 2009.
Sasmal, S. and Chakrabarti, S. K.: Ionosperic anomaly due to seismic activities Part 1: Calibration of the VLF signal of VTX 18.2 $\mathrm{KHz}$ station from Kolkata and deviation during seismic events, Nat. Hazards Earth Syst. Sci., 9, 1403-1408, doi:10.5194/nhess9-1403-2009, 2009.

Soloviev, O. V. and Hayakawa, M.: Three-dimensional subionospheric VLF field diffraction by a truncated high conducting cylinder and its application to Trimpi effect problem, Radio Sci., 37, 1079, doi:10.1029/2001RS002499, 639-647, 2002.

Soloviev O. V, Hayakawa, M., Ivanov, V. I., and Molchanov O. A.: Seismo-electromagnetic phenomenon in the atmosphere in terms of $3 \mathrm{D}$ subionospheric radio wave propagation problem, Phys. Chem. Earth, 29, 639-647, 2004.

Tobyas, V. and Mittag, R.: Local magnitude, surface wave magnitude and seismic energy, Studia Geophysica et Geodaetica, 35, 354-257, 1991.

Yoshida, M., Yamauchi, T., Horie, T., and Hayakawa, M.: On the generation mechanism of terminator times in subionospheric VLF/LF propagation and its possible application to seismogenic effects, Nat. Hazards Earth Syst. Sci., 8, 129-134, doi:10.5194/nhess-8-129-2008, 2008. 